\title{
Momento de la decisión estratégica: caso de estudio en el sector textil ${ }^{*}$
}

\author{
Strategic Decision Timing: A Case Study in the Textile Industry
}

\author{
Juan Esteban Hernández-Betancur \\ Doctor en Ingeniería - Industria y Organizaciones, Tecnológico de Antioquia Institución \\ Universitaria, Medellín - Colombia, juan.hernandez41@tdea.edu.co \\ Luz Alexandra Montoya-Restrepo \\ Doctora en Ciencias Económicas, Universidad Nacional de Colombia, \\ Medellín - Colombia, lamontoyar@unal.edu.co \\ Iván Alonso Montoya-Restrepo \\ Doctor en Ciencias Económicas, Universidad Nacional de Colombia, \\ Medellín - Colombia, iamontoyar@unal.edu.co
}

\begin{abstract}
Cómo citar / How to cite
Hernández-Betancur, J. E., Montoya-Restrepo, L. A., Montoya-Restrepo, I. A. (2022). Momento de la decisión estratégica: caso de estudio en el sector textil. Revista CEA, v. 8, n. 16, e1878. https://doi.org/10.22430/24223182.1878
\end{abstract}

Recibido: 7 de mayo de 2021

Aceptado: 23 de septiembre de 2021

\section{Resumen}

El objetivo de esta investigación fue realizar una aproximación organizacional en el momento de la decisión estratégica a partir de un caso de estudio en una empresa del sector textil del departamento de Antioquia (Colombia), haciendo uso de la integración interfuncional dentro del proceso de desarrollo de nuevo producto. Para ello se empleó una metodología basada en el proceso de construcción de conocimiento, realizado en la cual se aplicaron las herramientas de entrevista, proceso de observación y revisión de documentos de la empresa. Se evidenció, desde el caso, que el fenómeno del momento de la decisión, visto desde la aproximación organizacional de desarrollo de nuevo producto, involucra elementos como la integración interfuncional, la trayectoria cuasi tecnológica, los encuentros, las decisiones de exploración y explotación, el evento deconstructor, el agenciamiento, el trabajo conjunto, las directrices organizacionales, la información y el entorno. Se

\footnotetext{
* Este artículo se deriva del proyecto de investigación de la tesis doctoral titulada «Momento de la decisión estratégica: una mirada desde una aproximación organizacional a partir de las estrategias deliberadas y emergentes con el uso de simulación basada en agentes» y ha sido financiado con recursos de MinCiencias.
} 
hace pertinente replicar estos resultados en otras organizaciones e industrias, con el fin de ajustar y afianzar los mismos en el desarrollo de este caso.

Palabras clave: estrategia organizacional, colaboración interfuncional, gestión de la toma de decisiones, industria textil.

Clasificación JEL: L1, L67, M1, D81.

\title{
Highlights
}

- El momento de la decisión incide en el proceso de desarrollo de nuevo producto.

- Este estudio afianza la idea de que el momento de la decisión está constituido por la relación entre las estrategias deliberadas y emergentes.

- El momento de la decisión está asociado a elementos como la integración interfuncional, la trayectoria cuasi tecnológica, los encuentros entre agentes, las decisiones de exploración y explotación, el evento deconstructor, el agenciamiento, el trabajo conjunto, las directrices organizacionales, la información y el entorno.

\begin{abstract}
This paper investigates the timing of strategic organizational decision-making based on a case study of a textile company in the Department of Antioquia (Colombia) and cross-functional integration in the process of new product development. For this purpose, a methodology based on the knowledge construction process was adopted here applying three tools: interviews, observation, and review of company documents. In this case, it was found that the timing of the decision-making phenomenon, seen from the organizational perspective of new product development, involves elements such as cross-functional integration, quasi-technological trajectory, meetings, exploration-exploitation decisions, deconstructing event, agency, joint work, organizational guidelines, information, and environment. Further research should replicate these results in other organizations and industries in order to adjust and strengthen them.
\end{abstract}

Keywords: Organizational strategy, cross-functional collaboration, decision making management, textile industry.

JEL classification: L1, L67, M1, D81.

\section{Highlights}

- Decision timing influences the process of new product development.

- This study reinforces the idea that decision timing is defined by the relationship between deliberate and emergent strategies.

- Decision timing is associated with elements such as cross-functional integration, quasitechnological trajectory, meetings among agents, exploration-exploitation decisions, deconstructing event, agency, joint work, organizational guidelines, information, and environment. 


\section{INTRODUCCIÓN}

El problema de las decisiones ha sido abordado, principalmente, desde la perspectiva de encontrarle solución a una situación que necesita ser resuelta de este modo se busca la alternativa de solución que más se ajuste, según unos criterios de decisión predefinidos (Robbins y Coulter, 2005). Desde el aspecto anteriormente expuesto, se está afrontando la situación como un problema de contenido, pero también puede ser visto como un problema temporal, concentrado especialmente en las derivaciones de cuándo tomar la decisión (Smida, 2006; Chen y Tu, 2014). Esta segunda perspectiva del problema de la toma de decisiones ha sido relegada de los procesos de investigación, particularmente en lo referente a gestión estratégica (Sanabria Rangel, 2003; Smida, 2006; Tyagi et al., 2019). Más aun, las aportaciones teóricas son limitadas, contrario a las hechas desde la perspectiva práctica que, en algunos casos, son involuntarias o tangenciales (Hernández Betancur et al., 2016).

Teniendo en cuenta lo anterior, este documento que es pertinente comprender el fenómeno del momento de la decisión desde una aproximación organizacional. Para este caso, se decide observar el proceso de desarrollo de nuevo producto desde la perspectiva de la integración interfuncional con el fin de evidenciar una toma de decisiones continua, con elementos deliberados y emergentes en el contexto organizacional. Finalmente, poder contribuir a la explicación sobre las siguientes preguntas: ¿cómo es el funcionamiento del momento de la decisión estratégica desde una aproximación organizacional? ¿Cómo el proceso de formación de la estrategia y el ciclo que existe entre las estrategias deliberadas y emergentes pueden ayudar a la compresión del momento de la decisión estratégica desde una aproximación organizacional? ¿Qué variables se involucran en el proceso del surgimiento del momento de la decisión estratégica desde una aproximación organizacional? ¿Existe diferencia en el comportamiento del momento de la decisión en individuos, propuesto en desarrollos investigativos anteriores y desde una aproximación organizacional?

Para dar respuestas a las preguntas anteriormente expuestas, este artículo se encuentra distribuido de la siguiente manera: en primer lugar, se hace una revisión de literatura, donde se abordan los conceptos del momento de la decisión, se expone el proceso de desarrollo de nuevo producto desde la integración interfuncional como aproximación organizacional y algunas nociones que lo relacionan con el momento de la decisión. En segundo lugar, se enuncia la propuesta metodológica, que está centrada en el método de caso de estudio, propuesto por Eisenhardt (1989), donde se aplican los pasos de inicio, selección del caso y creación de instrumentos y protocolos, haciendo uso de las herramientas de entrevista, observación y revisión de documentos. En tercer lugar, se encuentran los resultados, en donde se despliegan los pasos de entrada en el campo y el análisis de los datos. En cuarto lugar, se cuenta con los pasos de la realización de hipótesis, envolvimiento de la literatura y el cierre, los cuales son expuestos en la discusión. Finalmente, en quinto lugar, se desenlaza el documento con las principales conclusiones. 


\section{MARCO TEÓRICO}

\section{Momento de la decisión}

Desde los estudios organizacionales, son escasas las contribuciones, algunas de ellas son las de Saavedra Mayorga (2006); Sanabria Rangel (2003); Smida (2006), quienes afirman que el momento de la decisión es una decisión en sí misma. Smida (2006) realiza una modelización del momento de la decisión, combinando tres tipológicas elementales; la primera de ellas que hace referencia a los momentos de decisión impuestos por el ambiente; la segunda, que habla de los momentos de decisión propuestos por el decisor; y la tercera, que indica los momentos de decisión posibles gracias a los recursos del decisor.

A su vez, Saavedra Mayorga (2006) y Helsing (2007), esbozan el momento de la decisión desde dos perspectivas, las cuales están enmarcadas en la cultura, siendo esta la dinamizadora del proceso de toma de decisiones. La primera de ellas está relacionada con respecto al procesamiento de información por parte del decisor para ponderar la alternativa más satisfactoria; la segunda está relacionada con hacer realidad la alternativa elegida.

Finalmente, Sanabria Rangel (2003) participa con contribuciones relacionadas con las decisiones de emergencia, cuando se presentan coyunturas sobre lo planeado y son necesarias decisiones no deliberadas, poniendo en discusión las rentas que se obtienen de la aplicación de decisiones, las cuales presentan variaciones dependiendo del momento en el cual se aplica.

Además de estas contribuciones, surgen las de Paul et al. (2005) y Greenstein (2015), quienes centran el problema de la decisión con relación al procesamiento de información. Los primeros discuten que la temporalidad de las decisiones en grupos de trabajo está centrada en la obtención y procesamiento de la información (Paul et al., 2005). El segundo plantea el equilibrio entre el tiempo que es gastado para procesar la información y en la manera como se afecta la función de utilidad de esa información mientras es procesada (Greenstein, 2015).

También se encuentran los aportes de Hernández Betancur et al. (2016), quienes enlistan las variables sustantivas e instrumentales sobre el momento de la decisión por medio de una revisión de literatura. Además, proponen que el momento de la decisión, en relación con las estrategias deliberadas y emergentes, surge cuando es deconstruida la estrategia deliberada y es requerido el surgimiento de la estrategia emergente, con el fin de que los agentes continúen siendo viables en el sistema para tomar decisiones (Hernández-Betancur et al., 2019).

\section{Aproximación organizacional a partir de la integración interfuncional}

Para realizar una observación directa del funcionamiento del momento de la decisión, se elige el proceso de desarrollo de nuevo producto. Las organizaciones deben atender los cambios constantes (Becerril-Elías y Merritt, 2021) de los consumidores de manera oportuna, situación recurrente en mercados dinámicos, en búsqueda de supervivencia en donde los ciclos de vida de los productos son cada vez más cortos, más aún en un contexto de competencia globalizada (Brown y Eisenhardt, 1995; Minguela Rata et al., 2000). 
Minguela Rata et al. (2000) y Marion y Fixson (2021) resaltan que el proceso de desarrollo de nuevo producto inicia con el sometimiento a evaluaciones de mercado, técnicas y financieras al grupo de ideas, con el fin de elegir las más apropiadas según las intenciones de la organización (Cooper y Kleinschmidt, 1986; Kotler y Keller, 2012). El proceso continúa con la determinación de características específicas del producto, con el fin de obtener un diseño preliminar (Kotler y Keller, 2012). En la siguiente etapa, se pretende convertir la idea y las características en elementos tangibles, condensados en prototipos, para comprobar el funcionamiento adecuado de los elementos del producto, poner a prueba la satisfacción del cliente, verificar los estándares de diseño y demás características puestas en la realidad (Cooper y Kleinschmidt, 1986; Minguela Rata et al., 2000; Kotler y Keller, 2012). Por último, está el diseño definitivo, etapa anterior al inicio de producción, en donde se tienen definidos los detalles del producto y se conocen las reacciones del mercado ante este (Cooper y Kleinschmidt, 1986; Minguela Rata al., 2000; Kotler y Keller, 2012).

Inmersa en el proceso de desarrollo de nuevo producto, se encuentra la integración interfuncional, en donde se espera una interacción constante del personal de diversos departamentos involucrados en el proceso (Ernst et al., 2010; Genç y Di Benedetto, 2015; Yongsheng y Jinjie, 2019). En la integración interfuncional aplican dos perspectivas. La primera está enfocada en el intercambio de información por parte de los departamentos de manera paralela y particularmente es señalada como conductual; la segunda está centrada en la concepción de la integración interfuncional como trabajo conjunto entre diferentes departamentos y que, puntualmente, se define como actitudinal (Ernst et al., 2010).

Urban y Hauser (1993) realizan una diagramación explícita de la integración interfuncional, involucrando los departamentos de Investigación y Desarrollo, Marketing, Producción y Finanzas dentro del proceso de desarrollo de nuevo producto, los cuales interactúan entre sí a partir de la definición de variables que los relacionan (ver Figura 1).

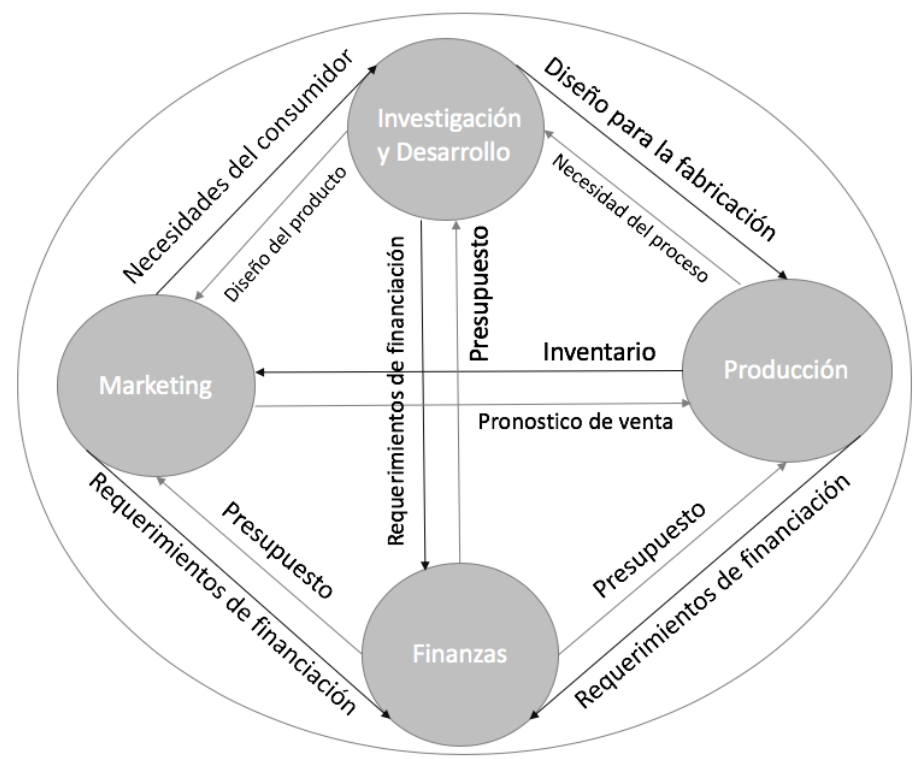

Figura 1: Integración interfuncional

Figure 1: Cross-functional integration Fuente: (Urban y Hauser, 1993) 
Inherentes al proceso de integración interfuncional y en el contexto de la definición del momento de la decisión se encuentran algunos conceptos que son fundamentales para el funcionamiento, los cuales son descritos a continuación.

\section{Algunos conceptos relacionados con la integración interfuncional y el momento de la decisión}

Se exponen los resultados de una revisión de literatura intencionada, en la búsqueda de concepciones teóricas relacionados con la integración interfuncional y el momento de la decisión.

\section{Sobre el momento de la decisión}

Estrategia emergente: la raíz del concepto de las estrategias deliberadas y emergentes se encuentra centrado en las aportaciones de Mintzberg $(1978,1994)$; Mintzberg y Waters (1985). Este concepto permite ampliar la discusión sobre la definición de la estrategia, la cual es bifurcada entre las estrategias deliberadas y emergentes. Las primeras concebidas como planes preestablecidos, y las segundas como construcción gradual a partir de las condiciones del entorno (Mintzberg, 1978). Se considera como elemento fundamental de la estrategia emergente el aprendizaje estratégico (Mintzberg y Waters, 1985). En Mintzberg (1994), se pretende darle valor a la estrategia emergente sobre la deliberada, resaltando las bondades de la primera en cuanto al aprendizaje, y en la segunda a los beneficios del control. Frente a lo anterior, Montoya Restrepo (2010); Montoya Restrepo y Montoya Restrepo (2013) desarrollan la idea de ciclo entre las estrategias deliberadas y emergentes.

Momento de la decisión: el momento de la decisión estratégica se toma principalmente desde la perspectiva que surge cuando la estrategia deliberada es deconstruida por un evento truncador que obliga al agente a buscar nuevas alternativas, constituidas en estrategias emergentes, que le permita continuar viable en el sistema para tomar decisiones futuras (Hernández Betancur et al., 2016).

El momento de la decisión participa en el proceso de desarrollo de nuevo producto, a partir de la deconstrucción de estrategias deliberadas por parte de los departamentos, las cuales no logran acoplarse con las de los demás y se hace necesario definir otras estrategias que permitan conservar la viabilidad. El momento de la decisión es definido cuando todos los departamentos están de acuerdo con sus respectivas variables.

Evento deconstructor: es considerado el impulsor de la estrategia emergente a partir de la inoperancia de la estrategia deliberada, lo que liga directamente a ese evento con el momento de la decisión. El evento deconstructor se define como un acontecimiento que produce discontinuidad en la simetría de los sucesos predefinidos dentro de los parámetros del espacio y el tiempo, teniendo un vínculo significativo con el manejo de la incertidumbre por parte del agente en las decisiones del pasado, presente y futuro (Hernández-Betancur et al., 2019).

Decisiones de exploración y explotación: las decisiones exploratorias responden a entornos inciertos, donde encaminan al agente a buscar alternativas con mayor frecuencia; por otro lado, las decisiones de explotación son propias de entornos catalogados como estables, donde el agente es llamado a sacar provecho de la información con la que cuenta, sin pensar en otras alternativas (Tzovara et al., 2012). 


\section{Sobre la integración interfuncional}

Integración interfuncional: se conceptualiza desde dos perspectivas; la primera, hace referencia a la capacidad que tienen departamentos funcionales en intercambiar información con otros de manera simultánea; la segunda, enfocada en la capacidad de trabajo conjunto alentado por una voluntad colectiva (Guo et al., 2017). Todo lo anterior, en el contexto de tener que desarrollar actividades colectivas que requieren de diferentes capacidades que son complementarias (Ernst et al., 2010).

A la discusión se suman Urban y Hauser (1993), proponiendo el modelo de integración interfuncional, constituido básicamente por los departamentos de Investigación y Desarrollo, Producción, Finanzas y Marketing, los cuales están relacionados a partir de ciertas variables en las cuales se espera que lleguen a acuerdos.

Cuasi trayectoria tecnológica: se realiza una transconcepción del concepto de trayectoria tecnológica definido para medir el crecimiento y evolución de una empresa, la cual se encuentra sumergida en una dinámica innovadora (Jasso, 2004).

La trayectoria tecnológica generalmente se encuentra constituida por tres etapas: crecimiento, madurez y declive (Jasso, 2004; Adamuthe y Thampi, 2019). Por el contexto del problema definido, se consideran las dos primeras etapas dentro del proceso de toma de decisiones, que incluye el desarrollo de nuevo producto, particularmente en la etapa de la idea.

Un aliciente a la decisión de usar el concepto de trayectoria tecnológica, para definir la construcción de la decisión conjunta de un grupo de departamentos para la elección de la idea en el desarrollo de nuevo producto, está centrada en que la medición de la trayectoria tecnológica depende, en gran medida, de la posición y el ritmo de la empresa con respecto al contexto innovador que caracteriza el proceso o producto que va a elaborar (Jasso, 2004), siendo, en este caso, los propios departamentos quienes otorgan esas variables características, con una posición y ritmo particular.

Además, se encuentra la dependencia de la trayectoria tecnológica con respecto a esfuerzo de otros agentes o del pasado, en cuanto a conocimientos, capacidades y recursos (Jasso, 2004), algo que se considera presente dentro de la integración interfuncional.

En relación con lo anterior, la cuasi trayectoria tecnológica implica el proceso que debe pasar para la construcción de la decisión de la idea dentro del proceso de desarrollo de nuevo producto a partir de la integración interfuncional, basada en la posición y el ritmo de los departamentos.

Encuentros (reuniones y acuerdos): para Demory (2007), las reuniones se pueden definir como el desarrollo de una tarea conjunta predefinida, dirigida principalmente por los participantes, los cuales fueron citados previamente.

Generalmente las reuniones están estructuradas por tres etapas: la primera, el inicio, en la cual quedan expuestos los temas y el objetivo que se van a tratar dentro de la reunión; el segundo es el núcleo, donde se amplían y discuten los temas tomando decisiones al respecto; y la última etapa es el resumen de los acuerdos logrados durante la interacción de la reunión (Muñoz Seco et al., 2018). 
Por otro lado, se encuentran las aportaciones sobre integración interfuncional, que son soportadas en las interacciones entre miembros de diferentes departamentos o áreas funcionales con el fin de construir acuerdos sobre decisiones en el proceso de desarrollo de nuevo producto (Ernst et al., 2010; Genç y Di Benedetto, 2015; Song y Parry, 1997; Urban y Hauser, 1993).

Agenciamiento: se puede definir genéricamente como "conjunto de relaciones cofuncionales entre elementos heterogéneos» (Heredia, 2014, p. 94). Desde la perspectiva organizacional se puede hablar de la construcción de conjunto o colectividad a partir de las unidades individuales asociadas a la adopción de una forma concreta de organización (Mintzberg y Van der Heyden, 2006).

Trabajo conjunto (cantidad de agentes por departamento): específicamente en la organización se dice que hay diversas manifestaciones que ejemplifican la superioridad de la inteligencia del grupo por encima de las de sus integrantes, poniendo en evidencia las bondades de las acciones coordinadas de los equipos; además, si se exponen a actividades de planificación en donde está presente de manera intrínseca la toma de decisiones, va a derivarse un aprendizaje de grupo excepcional (Senge, 1995; Zanazzi, 2009).

Directriz organizacional (cantidad de alternativas): con respecto a la definición de los objetivos, frente a la resolución de problemas en los procesos de toma de decisiones, se encuentra el hecho de identificar y seleccionar las alternativas deseables y posibles beneficiosas para darle solución al problema planteado dentro del proceso de toma de decisiones (Berumen y Llamazares Redondo, 2007). Desde esta perspectiva, directrices por los interesados en el proceso de toma de decisiones, pueden tener injerencias sobre la cantidad de alternativas, ya que logran participar en el acotamiento del problema.

Disponibilidad de información previa: existe una relación entre el procesamiento de la información y la función de utilidad de la solución elegida dentro de un proceso de toma de decisiones (Greenstein, 2015). Por su parte, Paul et al. (2005) y Citroen (2011) afirman que la información es fundamental para el proceso de toma de decisiones. Las decisiones que sufren retrasos de información tendrán implicaciones en cuanto a la calidad, ya que en decisiones grupales es relevante adquirir la información de manera temprana con el fin de tener tiempo suficiente para procesarla (Paul et al., 2005).

Entorno: corresponde a todos los elementos que son ajenos a la organización y que tienen consecuencia sobre sus decisiones futuras, en donde deben buscar entenderlos y adaptarse a ellos con el fin de garantizar su competitividad dentro de ese sistema (Sáez Vacas et al., 2003).

Según Sáez Vacas et al. (2003), los entornos organizacionales se pueden agrupar básicamente en tres tipos.

1. Entornos estables: se caracterizan por ser estables, simples, favorables e integrados.

2. Entornos adaptativos: son relativamente estables, algo complejos, prácticamente favorables y diversos.

3. Entornos inestables turbulentos: son dinámicos, complejos, hostiles y diversos. 


\section{METODOLOGÍA}

Se hace uso del caso de estudio, dado que es «una estrategia de investigación que se centra en la comprensión de la dinámica presente en una sola configuración» (Eisenhardt, 1989, p. 534). Además, otorga fortalezas como la novedad y validez empírica dada la vinculación íntima que tiene el investigador con la evidencia (Eisenhardt, 1989). Estos elementos son apropiados para abordar el problema del momento de la decisión teniendo en cuenta la característica del abandono de la temática que, por ende, ha sido poco desarrollado.

Para abordar el caso, con el objetivo de la generación de concepciones generales y contribuciones teóricas alrededor del momento de la decisión, se va a tener presente el proceso de construcción de teoría de la investigación del caso de estudio presentado por Eisenhardt (1989), el cual describe una serie de actividades que permiten dar desarrollo al caso hasta generar una saturación teórica con la información obtenida (ver Tabla 1).

Siguiendo los pasos definidos por Eisenhardt (1989), dentro del apartado de la Metodología se encuentran los pasos del inicio, la selección del caso y la creación de instrumentos y protocolos. Posteriormente, en los Resultados, se encuentran los pasos de la entrada al campo y el análisis de los datos. Finalmente, en la Discusión, se encuentran los pasos de haciendo hipótesis, envolviendo la literatura y el cierre.

Inicio

\section{Definición de las preguntas de investigación}

Desde el planteamiento de esta investigación se propusieron preguntas orientadas a responder de manera general el comportamiento del momento de la decisión estratégica desde una aproximación organizacional basada en el concepto de las estrategias deliberadas y emergentes. Ahora bien, sabiendo que la aproximación organizacional elegida va a estar centrada en el sector textil de Antioquia, teniendo en cuenta que es una industria dinámica frente al desarrollo de nuevos productos y diseños, que en consecuencia implican diversos procesos de toma de decisiones que no solo contiene elementos trascendentales de contenido de la decisión, sino que también de momentos de decisión, se reestructuran las preguntas de investigación, con el fin de definirlas específicamente para el caso de estudio, quedando de la siguiente manera.

\section{Pregunta General}

¿Cómo es el funcionamiento del momento de la decisión estratégica desde el proceso de desarrollo de nuevo producto en el sector textil antioqueño, particularmente desde la etapa de generación de ideas?

\section{Subpreguntas}

1. ¿Cómo el proceso de formación de la estrategia y el ciclo que existe entre las estrategias deliberadas y emergentes pueden ayudar a la compresión del momento de la decisión estratégica del proceso de desarrollo de nuevo producto, particularmente desde la etapa de generación de ideas? 
2. ¿Qué variables se involucran en el proceso del surgimiento del momento de la decisión estratégica desde el desarrollo de nuevo producto en el sector textil antioqueño, particularmente desde la etapa de generación de ideas?

\section{Constructos}

Los constructos, identificados de manera preliminar a partir de la literatura identificada, son los siguientes (ver Tabla 2).

Tabla 1: Proceso de construcción de la teoría de la investigación del estudio de caso Table 1: Process of building theory from case study research

\begin{tabular}{|c|c|c|}
\hline Paso & Actividad & Motivo \\
\hline Inicio & $\begin{array}{l}\text { Definición de la pregunta de } \\
\text { investigación. } \\
\text { Posibles constructos identificados } \\
\text { de manera a priori. }\end{array}$ & $\begin{array}{l}\text { Enfoca los esfuerzos. } \\
\text { Proporcionar una conexión aterrizada a partir de los } \\
\text { constructos. }\end{array}$ \\
\hline Selección del caso & $\begin{array}{l}\text { Ni teoría ni hipótesis. } \\
\text { Población especificada. } \\
\text { Muestreo teórico, no aleatorio. }\end{array}$ & $\begin{array}{l}\text { Conserva la flexibilidad teórica. } \\
\text { Restringe la variación extraña y agudiza la validez } \\
\text { externa. } \\
\text { Enfoca los esfuerzos en casos teóricamente útiles, es } \\
\text { decir, aquellos que replican o extienden la teoría } \\
\text { llenando categorías conceptuales. }\end{array}$ \\
\hline $\begin{array}{l}\text { Creación de } \\
\text { instrumentos y } \\
\text { protocolos }\end{array}$ & $\begin{array}{l}\text { Múltiples métodos de recolección } \\
\text { de datos. } \\
\text { Datos cualitativos y cuantitativos } \\
\text { combinados. } \\
\text { Múltiples investigadores. }\end{array}$ & $\begin{array}{l}\text { Fortalece la fundamentación de la teoría mediante la } \\
\text { triangulación de pruebas. } \\
\text { Visión sinérgica de la evidencia. } \\
\text { Fomenta perspectivas divergentes y refuerza las bases. }\end{array}$ \\
\hline Entrar en el campo & $\begin{array}{l}\text { Recopilación y análisis de datos } \\
\text { superpuestos, incluyendo notas de } \\
\text { campo. } \\
\text { Métodos de recogida de datos } \\
\text { flexibles y oportunistas. }\end{array}$ & $\begin{array}{l}\text { Acelera los análisis y revela ajustes útiles para la } \\
\text { recopilación de datos. } \\
\text { Permite a los investigadores aprovechar los temas } \\
\text { emergentes y las características únicas del caso. }\end{array}$ \\
\hline Análisis de datos & $\begin{array}{l}\text { Análisis dentro del caso. } \\
\text { Búsqueda de patrones cruzados } \\
\text { usando técnicas divergentes. }\end{array}$ & $\begin{array}{l}\text { Gana familiaridad con los datos y generación de teoría } \\
\text { preliminar. } \\
\text { Fuerza a los investigadores a mirar más allá de las } \\
\text { impresiones iniciales y ver pruebas a través de múltiples } \\
\text { lentes. }\end{array}$ \\
\hline Haciendo hipótesis & $\begin{array}{l}\text { Tabulación iterativa de la evidencia } \\
\text { para cada constructo. } \\
\text { Replicación, no muestreo, lógica } \\
\text { entre casos. } \\
\text { Buscar evidencia de "por qué" } \\
\text { detrás de las relaciones. }\end{array}$ & $\begin{array}{l}\text { Aumenta la definición, validez y mensurabilidad del } \\
\text { constructo. } \\
\text { Confirma, extiende y agudiza la teoría. } \\
\text { Crea validez interna. }\end{array}$ \\
\hline $\begin{array}{c}\text { Envolviendo la } \\
\text { literatura }\end{array}$ & $\begin{array}{l}\text { Comparación con la literatura en } \\
\text { conflicto. } \\
\text { Comparación con literatura similar. }\end{array}$ & $\begin{array}{l}\text { Crea validez interna, eleva el nivel teórico y agudiza las } \\
\text { definiciones de construcción. } \\
\text { Aumenta la generalización, mejora la definición de la } \\
\text { construcción y eleva el nivel teórico. }\end{array}$ \\
\hline Cierre & $\begin{array}{l}\text { Saturación teórica cuando es } \\
\text { posible. }\end{array}$ & $\begin{array}{l}\text { Termina el proceso cuando la mejora marginal se hace } \\
\text { pequeña. }\end{array}$ \\
\hline
\end{tabular}

Fuente: (Eisenhardt, 1989, p. 533). 
Tabla 2: Constructos del estudio de caso

Table 2: Constructs in this case study

\begin{tabular}{|c|c|}
\hline Constructo & Elementos que lo describe \\
\hline Estrategia emergente & $\begin{array}{l}\text { Ausencia de plan } \\
\text { No tiene una intención previa por parte del estratega, pero son } \\
\text { estrategias realizadas } \\
\text { Responde a características del entorno } \\
\text { Evoluciona de las estrategias deliberadas } \\
\text { Reorienta futuras estrategias deliberadas }\end{array}$ \\
\hline Momento de la decisión & Deconstrucción del plan y surgimiento de soluciones \\
\hline Integración interfuncional & $\begin{array}{l}\text { Interacción entre departamentos } \\
\text { Acuerdos entre variables de una decisión }\end{array}$ \\
\hline Cuasi trayectoria tecnológica & Posición y ritmo en la evolución de la idea \\
\hline Encuentros & Interacción entre agentes \\
\hline Decisiones de exploración & Buscar nuevas alternativas \\
\hline Decisiones de explotación & Aprovechar los beneficios de las alternativas ya conocidas \\
\hline Evento deconstructor & $\begin{array}{l}\text { Interrupción del flujo de decisiones } \\
\text { Origen endógeno o exógeno } \\
\text { Sorpresa o cambio abrupto o gradual } \\
\text { Disparador (negativo o positivo) }\end{array}$ \\
\hline Agenciamiento & Relación conjunta de agentes con diferentes características \\
\hline Trabajo conjunto & Cantidad de integrantes de los grupos de trabajo \\
\hline Directriz organizacional & Cantidad de alternativas \\
\hline Disponibilidad de la información & $\begin{array}{l}\text { Tiempo de llegada de la información } \\
\text { Volumen de la información }\end{array}$ \\
\hline Entorno & $\begin{array}{l}\text { Condiciones que lo convierte en un entorno: } \\
\text { Estable } \\
\text { Adaptativo } \\
\text { Turbulento }\end{array}$ \\
\hline
\end{tabular}

\section{Selección del caso}

\section{Población especificada}

Con la población que se va a trabajar especialmente dentro de este caso son los encargados y agentes involucrados dentro del proceso de desarrollo de nuevo producto, particularmente de la generación de la idea dentro de las empresas del sector textil, dedicadas a la manufactura en el departamento de Antioquia, en Colombia. El listado de empresas a tener en cuenta para identificar estas características es el de la Superintendencia de Sociedades (2019a, 2019b), en el cual están publicadas las empresas con mayor ingreso operacional del año 2018, clasificadas por sector, microsector y ubicación.

\section{Muestreo teórico}

En cuanto a la elección de la muestra, se decide comunicarse con cada una de las empresas del listado de la Superintendencia de Sociedades (2019a, 2019b), empezando por las que reportaron mayor ingreso operacional en ese periodo y proceder al contacto del personal responsable del desarrollo de 
nuevo producto, con el fin de obtener los permisos necesarios para aplicar los instrumentos de recolección de datos definidos para el caso. En la eventualidad que la empresa contactada no diera respuesta (o sea, negativa) a la invitación de participar en el estudio, se continuaba con la siguiente del listado.

Particularmente, para esta investigación, se decide realizar un caso de estudio único, partiendo de las aportaciones de Yin (2009), quien afirma que los casos de estudio único son útiles para verificar si proposiciones teóricas están conformes a la realidad o existen alternativas explicativas que se puedan identificar desde el caso.

\section{Instrumentos y protocolos}

Para esta investigación se decide contar con la opción de entrevista personal estructurada, observación directa no estructurada y revisión de documentos, teniendo en cuenta las opciones de recolección de información propuestas por Martínez Carazo (2006).

En cuanto a la entrevista personal estructurada se cuenta con un cuestionario que fue desarrollado a partir de los constructos propuestos (ver Anexo 1).

Por otro lado, con respecto a la observación, se pretende realizar directa no estructurada; la decisión de este tipo de instrumento parte de la característica novedosa de la perspectiva con la que se pretende observar el hecho; es decir que se considera un fenómeno relativamente nuevo el análisis al momento de elegir una idea dentro de un proceso de desarrollo de nuevo producto desde la perspectiva de las estrategias deliberadas y emergentes. Lo anterior tiene sustento en las sugerencias (Campos y Covarrubias y Lule Martínez, 2013), los cuales afirman que este tipo de observación es valiosa en investigaciones donde no se conoce mucho sobre el objeto de estudio.

Además, sugieren que la guía de observación debe contener como mínimo los siguientes datos (Campos y Covarrubias y Lule Martínez, 2013).

1. Datos y características de los sujetos a evaluar.

2. Propósitos de la observación o de las observaciones a realizar.

3. Temporalidad de la observación.

4. Lapsos o rangos de los momentos observables en apego a las categorías (constructos).

Finalmente, en cuanto a la revisión de documentos, se tiene acceso a diagramas de procesos relacionados al desarrollo de nuevo producto, a la página web corporativa y al canal de YouTube.

\section{RESULTADOS}

Como se indica en la metodología, en el apartado de Resultados se desarrollaron los pasos de: la entrada en el campo y el análisis de los datos, según lo planteado por Eisenhardt (1989), en donde se describió cómo fue la selección y visita a la empresa que participa del caso y cuáles fueron los resultados de aplicar los instrumentos. 


\section{Entrada al campo}

Para acceder a la empresa del caso de estudio se realizó una invitación a participar de la investigación por medio de una carta dirigida a los responsables de los procesos de desarrollo de nuevo producto. Se contactaron veinte empresas del sector textil del Valle de Aburrá, donde se obtuvo respuesta afirmativa por parte de una de las empresas, la cual se llamará Empresa ABC.

Empresa $A B C$ se dedicada a la exportación de prendas textiles desde la década de los noventa, ofreciendo a sus clientes un servicio especializado y empaquetado que incluye el diseño y desarrollo del producto, así como corte, confección, bordado, lavado, terminación y empaque. La compañía cuenta con unas instalaciones de 30000 metros cuadrados y aproximadamente 3000 empleados, distribuidos entre los procesos de terminación, lavandería, corte y confección. Actualmente, la compañía ofrece sus productos a clientes como Tommy Hilfiger, Levi's, Ralph Lauren, entre otros.

La visita a la empresa tuvo una duración aproximada de 90 minutos, en donde fue posible conocer el proceso productivo, desde el ingreso de materia prima, hasta el etiquetado del producto terminado; posteriormente se entrevistó al personal de Investigación y Diseño. Además, se accedió a los diagramas de proceso relacionados con la creación de muestras para clientes de exportación y documentación secundaria publicada en internet.

\section{Análisis de datos}

Como se indica en el apartado de Instrumentos y protocolos, se hace uso de tres herramientas: la primera de ellas es la entrevista, en donde en términos generales se indaga sobre el proceso de desarrollo de nuevo producto, los actores y funciones, restricciones temporales dentro del proceso, la participación de la información y la relación que tiene con las decisiones de exploración y explotación, cómo garantizan la interacción entre departamentos y cómo afrontan deconstrucción de los planes y el surgimiento de nuevas estrategias (ver Tabla 3).

Tabla 3. Respuesta entrevista caso de estudio

Table 3. Responses to the interview in this case study

\begin{tabular}{|c|c|}
\hline Pregunta & Respuesta \\
\hline $\begin{array}{l}\text { 1. Describa los pasos del proceso } \\
\text { de toma de decisiones de } \\
\text { desarrollo de nuevo producto. }\end{array}$ & $\begin{array}{l}\text { El proceso de desarrollo de producto dentro de esta empresa se divide } \\
\text { en dos, dependiendo si es una propuesta para el cliente o un } \\
\text { requerimiento del cliente. En la primera, la empresa realiza muestras } \\
\text { contenidas en un portafolio en donde le da a conocer todas las } \\
\text { capacidades con las que cuentan y que puedan ser de interés para el } \\
\text { cliente. Por otra parte, el segundo tipo de desarrollo de producto consiste } \\
\text { en atender directamente los requerimientos específicos del cliente, en } \\
\text { cuanto a diseño y costos de producción. }\end{array}$ \\
\hline $\begin{array}{l}\text { 1.1. ¿Qué actores participan en } \\
\text { esa toma de decisiones? }\end{array}$ & $\begin{array}{l}\text { El cliente, departamentos Comercial, de Diseño y de Desarrollo de } \\
\text { Producto. }\end{array}$ \\
\hline $\begin{array}{l}\text { 1.2. ¿Cuál es la función de cada } \\
\text { uno de esos actores? }\end{array}$ & $\begin{array}{l}\text { El cliente principalmente otorga las características del producto, en } \\
\text { cuanto al diseño, acabados y costos. } \\
\text { El Departamento Comercial es el encargado de mantener los niveles de } \\
\text { los costos, en las proporciones adecuadas para garantizar al cliente el } \\
\text { precio de venta. }\end{array}$ \\
\hline
\end{tabular}




\begin{tabular}{ll}
\hline Pregunta & \multicolumn{1}{c}{ Respuesta } \\
\hline & El Departamento de Diseño es el encargado conocer y entender la marca \\
& cliente, para poder ofrecerle las soluciones más satisfactorias en cuanto \\
& al diseño de las prendas. \\
& El Departamento de Desarrollo de Producto es el encargado de \\
& determinar los detalles de producción más adecuados para darle el \\
& acabado apropiado que quiere el cliente; por otro lado, es el encargado \\
& realizar las muestras para el cliente y que finalmente sea aprobada la \\
producción.
\end{tabular}

1.3. ¿ंDe los departamentos que participan en el proceso de desarrollo de la idea se involucran con uno o varios integrantes del departamento?

1.4. ¿Existen características particulares de comportamiento de los actores?

1.5. ¿Cuál es el resultado de la interacción entre los actores?

1.6. ¿Existe alguna restricción temporal de la acción de cada uno de los actores? Calendarios o cronogramas.

1.7. ¿Cómo es el proceso de elección de una idea?

1.8. ¿Existen directrices gerenciales o del grupo directivo frente a la generación de ideas? ¿Cuál es el efecto? ¿Pueden sentirse restringidos?

2. Participación de la información dentro del proceso de desarrollo de nuevo producto.

2.1 ¿Existe vigilancia del entorno para identificar elementos que afecte el proceso de toma de
Es un trabajo en equipo y todo el departamento está involucrado, pero siempre hay un líder por cada cliente.

Desarrollo de producto (producción): flexible. La empresa debe contar con varios métodos de producción, con el fin de ofrecer una versatilidad de acabados y diseños, teniendo un portafolio diverso para satisfacer las necesidades del cliente.

Diseño (informado): semiflexible. Conocer al cliente profundamente, lo que representa la marca y satisface a sus clientes, pero también tener la capacidad de ofrecerle propuestas innovadoras. Comercial (costos): garantizar el precio ofrecido al cliente.

El desarrollo del producto.

Desarrollo de Producto tiene una semana para definir el producto completamente, eso en cuanto a realización de las muestras que serán presentadas al cliente. La definición de la colección la hacen directamente en la interacción entre los departamentos de Comercial, Diseño y Desarrollo de Producto, lo que les toma una semana. La decisión de los clientes depende de la temporada y tienen periodos de antelación de la producción entre seis y doce meses, dependiendo de los clientes.

Básicamente parte de la interacción entre las áreas. El proceso de definición de las ideas es perfeccionado con el proceso de desarrollo de las muestras, en donde la comunicación entre las áreas y el cliente es fundamental.

La directriz de la empresa es sobre el cumplimiento de los requerimientos del cliente con altos estándares de calidad. Además hay una constante búsqueda de la eficiencia en cuanto a la producción, pues se espera siempre aumente la capacidad de la producción.

La información está involucrada constantemente dentro del proceso de desarrollo de nuevo producto, tanto recolectada de manera interna como externa. No solo se tiene en cuenta la información sobre el diseño, también sobre mejores prácticas de producción y conocimiento sobre el cliente.

Sí, fuentes de recolección interna o showroom (eventos y exposiciones de productos y capacidades), investigación de tendencias, plataformas digitales, briefs (documento con requerimientos de los clientes), conocimiento de la estética del cliente, ferias de moda, conocimiento 


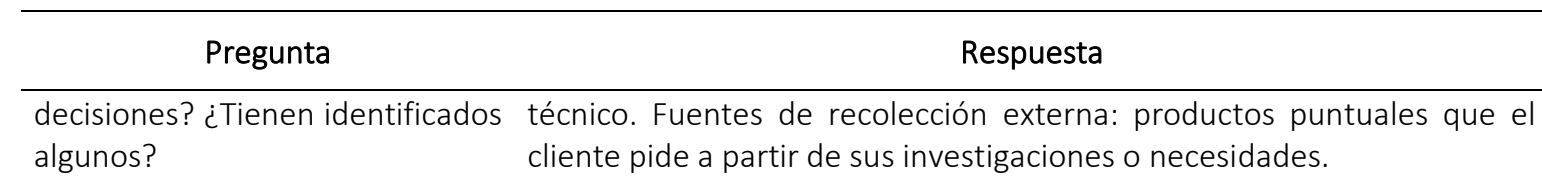

\begin{tabular}{|c|c|}
\hline $\begin{array}{l}\text { 2.2. ¿Han tenido desarrollos de } \\
\text { productos con innovaciones en } \\
\text { donde deban recolectar toda la } \\
\text { información requerida? }\end{array}$ & $\begin{array}{l}\text { Sí, cuando se les hace muestra de capacidades a los clientes para la } \\
\text { creación de demanda. }\end{array}$ \\
\hline $\begin{array}{l}\text { 2.3. ¿Qué diferencia se evidencia } \\
\text { frente al proceso de toma de } \\
\text { decisiones cuando existe } \\
\text { información previa y cuando no, } \\
\text { con respecto al proceso de } \\
\text { desarrollo de nuevo producto? }\end{array}$ & $\begin{array}{l}\text { Con la propuesta de creación de demanda se exhiben todas las } \\
\text { capacidades que tiene la empresa con el fin de encontrar un precio } \\
\text { objetivo de venta. En el caso de la petición de los clientes (cuando hay } \\
\text { información), todos los esfuerzos se concentran en satisfacer sus } \\
\text { necesidades, entre ellos el precio de la prenda. }\end{array}$ \\
\hline $\begin{array}{l}\text { 2.4. ¿La información previa invita } \\
\text { a explotar esas alternativas o a } \\
\text { explorar nuevas? }\end{array}$ & $\begin{array}{l}\text { En principio invita a explotar, dado que buscamos satisfacer las } \\
\text { necesidades de los clientes, pero exploran alternativas de solución ante } \\
\text { la búsqueda de la forma más efectiva de producción, eligiendo los } \\
\text { métodos más adecuados, por eso hacen pruebas de producción. }\end{array}$ \\
\hline $\begin{array}{l}\text { 3. ¿Qué estrategias aplican para } \\
\text { garantizar que los diferentes } \\
\text { departamentos interactúen entre } \\
\text { sí en el marco del proceso de } \\
\text { desarrollo de nuevo producto? }\end{array}$ & $\begin{array}{l}\text { Reuniones contantes, teniendo las líneas de comunicación abiertas. } \\
\text { Actualmente están realizando adecuaciones de un espacio de oficinas } \\
\text { para que queden en el mismo sector los departamentos de Comercial, } \\
\text { Diseño y Desarrollo de Nuevo Producto. }\end{array}$ \\
\hline
\end{tabular}

4. ¿Han afrontado situaciones dentro de la elección de la idea que deshace lo planeado? Constantemente dentro de la producción de la muestra identifican ¿Algunas veces esas situaciones algunos problemas como la calidad de los acabados y se toman los llevan a ideas viables y que no decisiones de cambio de máquinas donde se busca un acabado de mayor habían sido consideradas? calidad o eficiencia.

Estrategias deliberadas $y$ emergentes.

5. ¿ंDentro del proceso interdisciplinario de la generación de idea se identifica que hay departamentos que potencializan ideas por tener afinidad con ciertas alternativas? ¿Qué efecto tiene este tipo de alianzas?

Sí, esto genera aceleración del desarrollo de las ideas, porque todos están coordinados.

Fuente: elaboración propia.

La segunda herramienta es la observación directa no estructurada, la cual se realiza cuando se hace la visita por la planta de producción, conociendo todo el proceso productivo, desde la entrada de materia prima hasta el etiquetado del producto terminado, teniendo la oportunidad de observar específicamente el proceso de producción de muestras, muy vinculado al desarrollo de producto. Como mecanismo para la guía de observación se tiene en cuenta los constructos (ver Tabla 4). 
Los sujetos observados son todos los relacionados con el proceso de producción de la Empresa ABC, especialmente los del área de desarrollo de nuevo producto, la cual corresponde a la sección de la planta donde tiene una representación de toda la maquinaria que interviene en el proceso, pero a menor escala, con el fin del desarrollo de muestras para los clientes.

El propósito de la observación es identificar elementos que permitan corroborar la información suministrada dentro de la entrevista, que está asociada con la temporalidad de las decisiones dentro del proceso de desarrollo de nuevo producto. La observación es realizada durante 60 minutos, en los cuales se busca constantemente indicios observables de los constructos.

Tabla 4. Respuesta entrevista caso de estudio

Table 4. Observations in this case study

\begin{tabular}{|c|c|}
\hline Constructo & Observaciones \\
\hline $\begin{array}{l}\text { Estrategia } \\
\text { emergente }\end{array}$ & $\begin{array}{l}\text { El ciclo de producción de las colecciones depende del cliente, generalmente está establecido } \\
\text { de seis meses a un año. Es decir que algunas marcas tienen la producción con un año o seis } \\
\text { meses de antelación. Es probable que exista un ciclo entre estrategias deliberadas y } \\
\text { emergentes. Lo anterior significa que el concepto de moda que está en un periodo pasado } \\
\text { se convierte en tendencia para el ciclo siguiente y el nuevo concepto puede ser derivado de } \\
\text { la tendencia anterior y así sucesivamente. }\end{array}$ \\
\hline $\begin{array}{l}\text { Momento de } \\
\text { la decisión }\end{array}$ & $\begin{array}{l}\text { Se realizan prototipos de los productos con el fin de ir ajustando los acabados de las prendas } \\
\text { y tener muestras para los clientes. Se considera que en ese proceso se está construyendo el } \\
\text { momento de la decisión, en donde van surgiendo problemas con el plan, que son corregidos } \\
\text { por medio de estrategias emergentes. }\end{array}$ \\
\hline $\begin{array}{l}\text { Integración } \\
\text { interfuncional }\end{array}$ & $\begin{array}{l}\text { Existe interacción constante por parte de miembros de diferentes departamentos con el fin } \\
\text { de consolidar el desarrollo del nuevo producto. Durante la observación se presencia una } \\
\text { reunión entre personal de los departamentos de Desarrollo de Nuevo Producto y el } \\
\text { Comercial, discutiendo sobre la calidad de los acabados de un lote de muestra. Se intuye que } \\
\text { esta interacción concluye en acuerdos con base en las necesidades del consumidor y el } \\
\text { diseño del producto, como lo indica la integración interfuncional. }\end{array}$ \\
\hline $\begin{array}{l}\text { Cuasi } \\
\text { trayectoria } \\
\text { tecnológica }\end{array}$ & $\begin{array}{l}\text { En toda la planta de producción existe una buena distribución y ritmo de trabajo. Se } \\
\text { evidenció que hay un grupo de ingenieros encargados de medir tiempos y proponer métodos } \\
\text { de trabajo que aumente el ritmo de la producción. Con respecto al desarrollo de nuevo } \\
\text { producto, se están preocupando por la posición, ya que quieren generar una cercanía física } \\
\text { de los involucrados con el fin de crear mayor interacción. Pero la expresión más latente de } \\
\text { la cuasi trayectoria tecnológica es cuando construyen las muestras, porque es ahí donde se } \\
\text { ve reflejado de manera palpable todo el proceso de generación de la idea del nuevo } \\
\text { producto. }\end{array}$ \\
\hline Encuentros & $\begin{array}{l}\text { Los encuentros son constantes entre dependencias; de hecho se están realizando } \\
\text { adecuaciones físicas con el fin de generar una redistribución del personal dentro de la planta } \\
\text { para generar más fácilmente las interacciones entre los departamentos involucrados en el } \\
\text { desarrollo de producto. }\end{array}$ \\
\hline $\begin{array}{c}\text { Decisiones de } \\
\text { exploración }\end{array}$ & $\begin{array}{l}\text { Visitas de ferias de moda, visita de proveedores de maquinaria para renovación de procesos } \\
\text { y proponer nuevos acabados en las prendas }\end{array}$ \\
\hline $\begin{array}{l}\text { Decisiones de } \\
\text { explotación }\end{array}$ & $\begin{array}{l}\text { Creación de portafolios en los que se da a conocer a los clientes las capacidades que tiene } \\
\text { para ofrecer sus servicios, explotando lo que saben hacer. }\end{array}$ \\
\hline $\begin{array}{c}\text { Evento } \\
\text { deconstructor }\end{array}$ & $\begin{array}{l}\text { Se pueden generar eventos deconstructores dentro de la producción de la muestra, en } \\
\text { donde de manera endógena, por parte del personal de desarrollo de producto o por parte } \\
\text { del cliente, es decir exógeno, pueden sugerir cambios en los acabados que implica }\end{array}$ \\
\hline
\end{tabular}




\begin{tabular}{|c|c|}
\hline Constructo & Observaciones \\
\hline & $\begin{array}{l}\text { modificaciones de la producción. Generalmente son cambios que saben que van a pasar y } \\
\text { que surgen de marea gradual, los cuales son discutidos habitualmente entre Desarrollo de } \\
\text { Producto, Comercial y Diseño. }\end{array}$ \\
\hline Agenciamiento & $\begin{array}{l}\text { Durante el tiempo de la observación no se captó una muestra significativa de agenciamiento, } \\
\text { pero sí se evidenció un buen clima laboral, el cual puede facilitar ese proceso de } \\
\text { relacionamiento entre agentes diferentes. Además, se observó contacto constante ente } \\
\text { departamentos. }\end{array}$ \\
\hline $\begin{array}{l}\text { Trabajo } \\
\text { conjunto }\end{array}$ & $\begin{array}{l}\text { En el área de Desarrollo de Producto se observó una persona de Comercial con personal de } \\
\text { Desarrollo de Nuevo Producto teniendo una conversación sobre los acabados de una } \\
\text { muestra. }\end{array}$ \\
\hline $\begin{array}{c}\text { Directriz } \\
\text { organizacional }\end{array}$ & $\begin{array}{l}\text { Garantizar a los clientes los productos con calidad y a tiempo, constantemente, sobre todo } \\
\text { en producción, pues es allí donde se hacen revisiones de métodos y tiempos en búsqueda } \\
\text { de tener eficiencia en la producción. Además, tiene varios puntos de control de calidad y un } \\
\text { área exclusiva de corrección de esos defectos, con el fin de entregar la mejor calidad del } \\
\text { producto. De igual forma se da una acotación de alternativas por medio del seguimiento de } \\
\text { las necesidades del cliente y las disposiciones organizacionales. }\end{array}$ \\
\hline $\begin{array}{l}\text { Disponibilidad } \\
\text { de la } \\
\text { información }\end{array}$ & $\begin{array}{l}\text { Dentro de la producción de las muestras de nuevo producto, los operarios cuentan con una } \\
\text { muestra de los acabados. } \\
\text { En la sala de reuniones cuentan con un showroom y muestra de las telas. } \\
\text { Revisión constante del significado de las marcas para ofrecerle conceptualmente el mejor } \\
\text { proceso de producción que se adapte a ese significado. }\end{array}$ \\
\hline Entorno & $\begin{array}{l}\text { Entorno relativamente estable en cuanto a la confección de prendas de vestir tradicionales, } \\
\text { pero con contenido de moda que es llamativo en el mercado. Tiene clientes claramente } \\
\text { definidos y cuentan con una marca propia }\end{array}$ \\
\hline
\end{tabular}
Fuente: elaboración propia.

Finalmente, el tercer instrumento usado dentro del caso de estudio es la revisión de documentos. Para este caso se hace uso de diagramas de proceso relacionados con la creación de muestras para los clientes. Además, de forma complementaria, también se tomó información de la página web de la empresa y de videos corporativos del canal de YouTube. Al igual que en la observación, se toman los constructos del caso como referencia en búsqueda de hallazgos en la información secundaria (ver Tabla 5).

Tabla 5. Resultados revisión de documentos

Table 5. Results of the document review

\begin{tabular}{cl}
\hline Constructo & \multicolumn{1}{c}{ Hallazgos } \\
\hline & $\begin{array}{l}\text { Soluciones a los cambios y recomendaciones que hace el cliente permanentemente dentro } \\
\text { del proceso. }\end{array}$ \\
Estrategia & $\begin{array}{l}\text { Revisando la historia de la empresa se denota un emprendimiento emergente, que surge de } \\
\text { emergente } \\
\text { la deconstrucción de la declaración en bancarrota de otra empresa, donde es conseguida la } \\
\text { maquinaria, empleados y algunos clientes. En videos institucionales afirman que realizan } \\
\text { proceso de reinvención constante a partir de las mismas herramientas con las que cuenta. }\end{array}$ \\
\hline $\begin{array}{c}\text { Momento de } \\
\text { la decisión }\end{array}$ & $\begin{array}{l}\text { Dentro del proceso de definición del target se realizan ajustes a las muestras del cliente, lo } \\
\text { que implica una deconstrucción de planes y surgimiento de nuevas estrategias. Esta } \\
\text { situación también se presenta en la definición de colores según las pretensiones del cliente. }\end{array}$ \\
\hline
\end{tabular}




\begin{tabular}{|c|c|}
\hline Constructo & $\begin{array}{ll}\text { Hallazgos } \\
\end{array}$ \\
\hline $\begin{array}{l}\text { Integración } \\
\text { interfuncional }\end{array}$ & $\begin{array}{l}\text { En los diagramas de proceso se evidencia que el Departamento de Desarrollo se comunica } \\
\text { frecuentemente con las áreas Textil, Comercial y Diseño para aclarar o notificar aspectos de } \\
\text { la producción. Esto da indicios de trabajo conjunto entre diferentes departamentos. }\end{array}$ \\
\hline $\begin{array}{l}\text { Cuasi } \\
\text { trayectoria } \\
\text { tecnológica }\end{array}$ & $\begin{array}{l}\text { Los diagramas de procesos en sí mismo son una representación de la construcción de la cuasi } \\
\text { trayectoria tecnológica del desarrollo de nuevo producto, en donde se deja claro el ritmo de } \\
\text { construcción de la idea. }\end{array}$ \\
\hline Encuentros & $\begin{array}{l}\text { Como se indica en el constructo de la Integración internacional, los diagramas de proceso } \\
\text { sugieren en algunas situaciones interacciones con otras áreas, las cuales, de alguna forma, } \\
\text { se dan como encuentros. }\end{array}$ \\
\hline $\begin{array}{l}\text { Decisiones de } \\
\text { exploración }\end{array}$ & $\begin{array}{l}\text { Dentro del proceso tienen la posibilidad de usar telas que no son conocidas y las adicionan } \\
\text { al listado de telas disponibles. Dentro de videos institucionales invitan a explorar alternativas } \\
\text { futuras, de manera global de la empresa. }\end{array}$ \\
\hline $\begin{array}{l}\text { Decisiones de } \\
\text { explotación }\end{array}$ & $\begin{array}{l}\text { En sí el diagrama de proceso es una decisión de explotación, dado que tiene establecido paso } \\
\text { a paso el proceso del desarrollo de prototipos de producto según los parámetros de cliente } \\
\text { internacional. En videos institucionales, al igual que invitan a explorar nuevas alternativas, } \\
\text { también invitan a explotar el hoy. }\end{array}$ \\
\hline $\begin{array}{c}\text { Evento } \\
\text { deconstructor }\end{array}$ & $\begin{array}{l}\text { Dentro del proceso se pide que se deben dejar constancia de los cambios que vayan } \\
\text { afectando el precio target. }\end{array}$ \\
\hline Agenciamiento & $\begin{array}{l}\text { El agenciamiento es posible dentro de los diagramas de proceso, porque el trabajo conjunto } \\
\text { está eventualmente presente dentro del desarrollo de nuevo producto. No queda explícito } \\
\text { dentro del diagrama, pero está la posibilidad de ocurrencia de este fenómeno. }\end{array}$ \\
\hline $\begin{array}{l}\text { Trabajo } \\
\text { conjunto }\end{array}$ & $\begin{array}{l}\text { Dentro del proceso trabajan varios departamentos, tales como Desarrollo de Producto, el } \\
\text { cliente, las áreas Textil, Comercial, Diseño (entrega las características de los acabados } \\
\text { cuando es un diseño propio) e Investigación y Desarrollo (entrega fichas técnicas y } \\
\text { estandarización, y asesora, si es necesario, en el desarrollo del proceso). }\end{array}$ \\
\hline $\begin{array}{c}\text { Directriz } \\
\text { organizacional }\end{array}$ & $\begin{array}{l}\text { En sí mismo el diagrama de proceso es una directriz organizacional, en donde se evidencia } \\
\text { el interés por cumplir los requerimientos del cliente y tener una interacción constante entre } \\
\text { las diferentes áreas, dejando clara la información. }\end{array}$ \\
\hline $\begin{array}{l}\text { Disponibilidad } \\
\text { de la } \\
\text { información }\end{array}$ & $\begin{array}{l}\text { Hay constante comunicación entre las diferentes áreas; por ejemplo, si es un nuevo } \\
\text { desarrollo, el Área de Desarrollo le debe consultar al Área Textil el fabric profile para definir } \\
\text { las condiciones de los acabados. Por otro lado, Investigación y Desarrollo entrega fichas } \\
\text { técnicas y estandarización. } \\
\text { Desarrollo de Producto realiza el manual de la unidad, basado en las directrices del jefe de } \\
\text { cuenta y lo descrito en la orden de trabajo y entrega la ficha de la prenda a las áreas } \\
\text { involucradas. }\end{array}$ \\
\hline Entorno & $\begin{array}{l}\text { No hay evidencia del entorno en el diagrama del proceso. Pero en videos institucionales } \\
\text { reiteran que la empresa es una de las principales exportadoras de textiles del país y que hace } \\
\text { parte de una industria que tiene un grado de complejidad y es muy competitivo } \\
\text { internacionalmente. Además, que hace parte del negocio de la moda, el cual es } \\
\text { constantemente cambiante. }\end{array}$ \\
\hline
\end{tabular}

Fuente: elaboración propia.

\section{DISCUSIÓN}

Este apartado se fundamenta en los últimos tres pasos de la metodología de Eisenhardt (1989) para casos de estudio; primero se plantean hipótesis a partir de los resultados obtenidos anteriormente y luego se realiza una discusión generando contraste con las teorías anteriormente planteadas en el envolvimiento de la literatura y cierre. 


\section{Haciendo hipótesis}

En cuanto al concepto de estrategia emergente, dentro de las respuestas de la entrevista, se puede identificar que la compañía, en el proceso de desarrollo de producto, debe atender recurrentemente modificaciones de la idea, ya que existe una variedad considerable de tipos de acabados y detalles dentro del desarrollo de las prendas, los cuales pueden ser requeridos por el cliente o sugeridos por la compañía; esto es confirmado por la revisión de documentos, donde en los diagramas de procesos se tiene establecida la actividad de soluciones a cambios y recomendaciones que hace el cliente. Además, en las indagaciones dentro de la observación se identifica que los ciclos de producción, requeridos por los clientes, son aproximadamente entre seis meses y un año, en donde las marcas tienen fabricadas las prendas con ese tiempo de antelación, lo que indica una línea directa entre las tendencias de moda y las colecciones.

Teniendo en cuenta lo anterior, se evidencia que, a partir de la necesidad de la estrategia emergente, se hace presente el concepto del momento de la decisión, puntualmente en las situaciones en donde lo planeado deliberadamente debe ser revisado y ajustado. En particular esta situación en los documentos se identifica en los diagramas de proceso en la definición del target o características del producto, los cuales deben ser validados por el cliente. Esto fue aprobado en la observación, donde se evidencia un espacio exclusivo en la planta para la producción de muestras para clientes con el fin de validar las características del producto.

Con respecto a la integración interfuncional, se evidencia dentro de la entrevista que es fundamental en el proceso de toma de decisiones en la elección de la idea, esto basado en la interacción de departamentos que tienen injerencia en el proceso de desarrollo de nuevo producto, ajustando características de este. Es posible corroborar esto en la observación y en la revisión de documentos, ya que en la primera fue posible observar la interacción de miembros de diferentes departamentos ajustando características del producto, y en la segunda, en el diagrama de proceso, queda explícita la intervención de varias áreas de trabajo dentro del proceso.

Referente al concepto de cuasi trayectoria tecnológica, se convierte en la evidencia dinámica de la integración internacional, la cual se puede ver plasmada en los diagramas de proceso, los cuales son una evidencia gráfica de cómo se construye la cuasi trayectoria tecnológica del producto por medio de integración interfuncional. Además, dentro de la observación se pudo constatar interés por parte de la organización en cuanto a la distribución (posición) y métodos y tiempos de trabajo (ritmo) del desarrollo de los productos.

La interacción entre los departamentos para la integración interfuncional se da a partir de encuentros, específicamente en la observación se puedo ver que se dan interacciones entre miembros de diferentes departamentos con el fin de generar acuerdos con respecto a características del producto. A lo anterior, se suman las adecuaciones que estaban realizando, al momento de la visita, en la zona de oficinas para tener una cercanía física mayor ente los departamentos que participan del proceso de desarrollo de nuevo producto con el fin de facilitar las interacciones. Estos encuentros también son considerados dentro de los diagramas de proceso.

En cuanto a las decisiones de exploración y explotación, desde la entrevista se identifica un uso mixto de estas, ya que explotan las decisiones que compone la estrategia de satisfacer a cabalidad las 
necesidades del cliente, pero a su vez implementan decisiones de exploración buscando alternativas más eficientes de producción, con acabados novedosos y versatilidad en las posibilidades de propuestas para los clientes. Esto es corroborado en la observación, donde se evidencia la explotación de las capacidades adquiridas en la oferta de diferentes acabados de producción a los clientes, pero también la constante exploración en ferias de moda y herramientas de producción con el fin de ofrecer nuevos acabados dentro del producto. En cuanto a la revisión de documentos, particularmente de los diagramas de proceso, se evidencia la posibilidad de incorporación de nuevas materias primas, según las necesidades del diseño, lo que permite, eventualmente, la aplicación de decisiones de exploración. En cuando a las decisiones de explotación, los diagramas de proceso en sí mismo denotan la aplicación de una alternativa que es satisfactoria.

En cuando al concepto del evento deconstructor, se puede esbozar desde la entrevista que está presente recurrentemente en la construcción de muestras y validación por parte del cliente, en donde constantemente se deben encontrar alternativas para solucionar dicha situación; esto se puede corroborar con la observación, con las conversaciones por parte de personal de Comercial y de Desarrollo de Producto en la zona de Calidad, en donde se verifica el cumplimiento del estándar que quieren del acabado. En la revisión de documentos, particularmente en el diagrama de proceso, se identifica que no descarta la posibilidad de que existan cambios que puedan afectar el precio objetivo del producto y que tienen que estar debidamente documentados.

Con respecto al agenciamiento, no fue posible evidenciarlo dentro de la observación y la revisión de documentos. Pero durante la entrevista manifiestan que, en algunos casos, sí se hace presente y eso acelera el proceso de desarrollo de nuevo producto, ya que implica una coordinación por parte de todos los departamentos que participan del proceso.

El otro concepto es el trabajo conjunto, del cual, a partir de la entrevista, se logra vislumbrar a las reuniones y la comunicación directa como mecanismos de interacción entre los departamentos de Comercial, Diseño y Desarrollo de Producto; de hecho, están en proceso de reformas en la infraestructura de las oficinas, con el fin de poder generar una aproximación física mayor entre los departamentos involucrados en el proceso de desarrollo de producto. Particularmente con la herramienta de observación se corrobora ese proceso de interacción dentro del área de Fabricación de Muestras de la zona de Lavandería, donde personal del departamento de Comercial sostenía conversaciones con personal de Desarrollo de Producto sobre los acabados de unas muestras en particular. Por su parte, dentro de los documentos se puede identificar la participación de los departamentos de Textil, Desarrollo de Producto, Comercial y Diseño, revelando cuál es su interacción a partir de los entregables entre ellos. Es de anotar que otro actor que es resaltado dentro del proceso de desarrollo de producto es el cliente, otorgando directrices en cuanto a características y costos del producto.

Con respecto al concepto de directriz organizacional, en los tres instrumentos del caso de estudio, se identifica que la organización tiene la pretensión de cumplir los requerimientos de los clientes con altos estándares de calidad. Otro actor importante que otorga directrices es el cliente, el cual está en constante contacto con la empresa, a su vez que la compañía hace un trabajo considerable en tener el suficiente conocimiento sobre el estilo y tendencias de la marca. 
Referente a la disponibilidad de información, en la entrevista se evidencia la importancia de esta dentro del proceso de desarrollo de nuevo producto, como por ejemplo buscar comprensión del cliente para darle las opciones de diseño y acabados que se ajusten a sus necesidades y tener las capacidades de producción apropiadas para incentivar la demanda. En la observación se identifica la presencia de varios catálogos de muestras para los clientes, tanto así que la sala de reuniones está constituida como un showroom en donde exhiben varias prendas. Además, en la revisión de documentos, queda explicito el uso de la información interna y como viaja a través de los diferentes departamentos involucrados en el desarrollo de nuevo producto.

Finalmente, con respecto al concepto del entorno, en la entrevista la compañía menciona que cuenta con dos fuentes de comprensión del entorno, la primera de ellas es de recolección interna, donde hace esfuerzos de participar en showroom, investigación de tendencias, ferias de moda, conocimiento de la estética del cliente, conocimiento técnico y plataformas digitales, en donde pretenden construir la dinámica reciente del entorno con el fin de tener las herramientas necesarias para responder a él. La segunda es por fuente de recolección externa, la cual está enfocada en los productos que el cliente puntualmente requiere y donde él mismo realiza sus propias investigaciones o identifica las necesidades que lo atañe. En la observación se tiene la percepción de que la compañía se encuentra en un entorno relativamente estable, con un producto y un mercado definidos, pero en constante renovación de capacidades para responder a las exigencias que se puedan presentar. Por su parte, en la revisión de documentos se da a entender que la organización cuenta con un posicionamiento de sus productos ante marcas internacional, pero que a su vez es una industria con complejidades y que es muy competitiva en el ámbito internacional, el cual está en constante cambio.

\section{Envolviendo la literatura}

En este apartado, con la información y la teoría expuestas anteriormente, se pretende realizar el envolvimiento de la literatura y cierre del caso de estudio, dándole repuesta a los dos últimos pasos propuestos por Eisenhardt (1989).

Con respecto al proceso de formación de la estrategia, según lo propuesto por Mintzberg y Waters (1985), Mintzberg (1978; 1987), Jørgensen y Mintzberg (1987), Mintzberg y McHugh (1985), de manera emergente se deben atender los requerimientos del cliente para continuar el camino hacia la estrategia pretendida, que en este caso particular se establece en precios objetivos o calidad de acabados basados en un diseño preestablecido. Por otro lado está la relación cíclica entre las estrategias deliberadas y emergentes propuestas por Montoya Restrepo (2010), Montoya Restrepo y Montoya Restrepo (2013), la cual es evidente en los ciclos de generación de colecciones y la anticipación que requieren, ya que una colección es producida aproximadamente con una diferencia entre seis meses y un año de antelación, lo que implica que las tendencias de moda que son usadas para la generación (estrategias deliberada) sean alimentadas de modificaciones dentro del proceso de producción (estrategias emergentes), de las cuales, en principio, se basarán colecciones futuras y así reiterativamente.

Referente al momento de la decisión, se tiene en cuenta las aportaciones de Hernández-Betancur et al. (2019), las cuales expresen que el momento de la decisión corresponde al surgimiento de la estrategia emergente ante la deconstrucción de la estrategia deliberada. Dentro del caso, esto se hace evidente en el proceso de desarrollo de nuevo producto cuando se considera que es necesario 
revisar algunos aspectos del proceso como algunas características del producto, en donde deben surgir estrategias emergentes que propongan soluciones satisfactorias y permitan la continuidad del proceso de desarrollo de nuevo producto.

Con relación a la integración interfuncional, se evidencia la interacción entre diferentes departamentos con el fin de intercambiar información, como lo plantean desde la perspectiva teórica Ernst et al. (2010), Genç y Di Benedetto (2015), Song y Parry (1997). Además, el hallazgo de la participación en el proceso de desarrollo de nuevo producto de departamentos como Diseño, Comercial y Producción, muy cercanos estos a los planteados por Urban y Hauser (1993), Investigación y Desarrollo, Producción, Marketing y Finanzas.

En cuanto a la cuasi trayectoria tecnológica, desde Jasso (2004), se toman el concepto de evolución y madurez de una empresa para incorporar en el proceso de generación de una idea dentro del desarrollo de nuevo producto. Esta concepción es evidente dentro del caso. Se podría decir que los diagramas de proceso en sí mismo se convierte en una forma de plasmar la trayectoria tecnológica de la idea. Por otro lado, están los mecanismos de medición de la trayectoria tecnológica, que son la posición y el ritmo, algo que en el caso es evidente en cuanto a la distribución en planta y oficinas y el estudio de métodos y tiempos de trabajo que ayuden a la velocidad y coordinación de las actividades.

Como un aspecto natural de la integración interfuncional existen los encuentros, los cuales permiten la interacción entre los departamentos, algo que fue evidente dentro del caso, pudiendo observar una reunión entre miembros de departamentos diferentes. Desde el ámbito teórico, las reuniones o encuentros son definidos como el desarrollo de tareas conjuntas (Demory, 2007), los cuales desenlazan en acuerdo generados a partir de la interacción dentro del encuentro (Muñoz Seco et al., 2018).

Con respecto a las decisiones de exploración y explotación, se pudo detectar una constante combinación del uso de ellas, en algunas situaciones explotan alternativas conocidas, pero sin descartar explorar nuevas soluciones que traigan beneficios superiores. Desde la teoría se encuentra que Hernández Betancur et al. (2016) postula a las decisiones de exploración y explotación como una variable sustantiva del momento de la decisión. Las decisiones de exploración, en el sentido de buscar alternativas que permitan obtener mejores resultados en proceso de toma de decisiones, y las de explotación, sacando provecho a alternativas conocidas que no entregan el mejor resultado, pero ayuda a la velocidad con la que se toman las decisiones (Tzovara et al., 2012).

Desde el punto de vista teórico, el concepto del evento deconstructor, determinado en el contexto del momento de la decisión por Hernández-Betancur et al. (2019), es visto como un acontecimiento que genera una ruptura en la simetría de eventos preestablecidos en forma de estrategia deliberada en un contexto de espacio y tiempo, el cual detona la necesidad de surgimiento de una estrategia emergente. En el caso, este evento deconstructor está presente, en cierta medida, en el proceso de desarrollo de nuevo producto, cuando deben realizar cambios en las características del producto a partir de requerimientos del cliente.

Referente al agenciamiento, fue un concepto difícil de identificar dentro del caso; sin embargo, en la entrevista se obtuvo la declaración que en algunos casos se hacía presente como un colectivo que es 
beneficioso para la aceleración del desarrollo de nuevo producto. Desde la teoría, el concepto de agenciamiento es definido por Heredia (2014) como agentes diversos que tienen un grupo de relaciones y funciones comunes, que, desde el contexto organizacional, se puede entender como un grupo de unidades individuales asociadas que toman la forma de organización en búsqueda de un objetivo común (Mintzberg y Van der Heyden, 2006).

En cuanto al trabajo conjunto, los aspectos identificados en el caso de estudio son comparables con la integración interfuncional propuesta por Urban y Hauser (1993), a pesar de que los departamentos no son nombras exactamente iguales, se resalta la participación de diferentes áreas responsables de diversas funciones que son fundamentales para que se desarrolle el nuevo producto. Además, se identifica la participación fundamental de la interacción y la comunicación dentro del proceso de integración, como lo indica Ernst et al. (2010), donde se pone en evidencia la superioridad de la inteligencia del grupo por encima de las individuales para tomar decisiones y resolver problemas (Senge, 1995; Zanazzi, 2009).

Por otro lado, relacionado a la directriz organizacional, se evidencia que se aproxima a las aportaciones de Maritz et al. (2011) en cuanto al liderazgo gerencial de la estrategia emergente, las cuales implican adaptación a contextos cambiantes, con respuestas adaptativas y rápidas a decisiones y acciones más autónomas, representadas en los cambios solicitados por los clientes y la respuesta versátil que se ofrece desde el departamento de Desarrollo de Producto, entregando una respuesta veloz a la situación.

A su vez, la información en el caso de estudio se resalta considerablemente, siendo relevante en cuanto a la relación de la compañía con los clientes y el entorno, otorgando la capacidad de generar alternativas por medio de los catálogos de muestra, dándole a conocer al mercado las capacidades con las que cuenta. Por otro lado, en la literatura se identifican las aportaciones de (Paul et al., 2005), quienes centran la discusión de la relación entre el tiempo y la decisión en la consecución y procesamiento de información de grupos de trabajo, donde estos reciben información tardía, pero toman decisiones más rápidas que los grupos que reciben la información más temprana. Por su parte, Zhang y Bei (2006), llaman la atención sobre la necesidad de que los estrategas de la organización deben tener la capacidad de equilibrar la adquisición de la información y el aprovechamiento de las posibilidades y oportunidades de decisión. A su vez, Good (2014) resalta que la capacidad de adaptación al entorno de las compañías requiere compresión del ambiente, modificar la trayectoria cuando sea necesario y concentrarse en la información relevante. En la discusión de la información también participa Greenstein (2015), el cual afirma que el mejor momento para tomar las decisiones se encuentra en el equilibrio del manejo de la presión del tiempo para adquirir la información, pero también la afectación de la utilidad que sufre con el tiempo. Finalmente, están las aportaciones de Hernández Betancur et al. (2016), quienes vinculan a la información como una variable sustancial e instrumental del concepto del momento de la decisión.

Por último, con respecto al entorno, desde las aportaciones de Sáez Vacas et al. (2003), son los elementos fuera de la organización que tiene efectos sobre las decisiones futuras, donde es necesario entenderlos y adaptarse para garantizar la viabilidad. Los entornos se pueden clasificar en estables, adaptativos y turbulentos. Desde el punto de vista del caso se evidencia vigilancia del entorno, haciendo una revisión constante del mercado por medio de ferias textiles y seguimiento de las tendencias de los clientes. 


\section{Cierre}

A manera de síntesis, se puede enunciar que el proceso de formación de la estrategia está presente en la aproximación organizacional de desarrollo de nuevo producto, donde hacen concurrencia las estrategias deliberadas con el análisis de los clientes y propuestas por parte de la empresa para atender sus colecciones. Las estrategias emergentes también son visibles en cuanto a generar soluciones ante las solicitudes de cambios por parte de los clientes, en donde pueden ser usados acabados de colecciones pasadas, denotando el uso de una estrategia deliberada anterior, ahora usada como una estrategia emergente, dando a entender la presencia del ciclo. En este sentido, la visión del momento de la decisión es evidente cuando se deben tomar decisiones sobre esos cambios dentro de las colecciones.

También queda en evidencia que la construcción de las estrategias emergentes, dentro del desarrollo de nuevo producto, hace parte de la integración interfuncional entre los diferentes departamentos que participan, básicamente con la interacción en encuentros, establecidos en un trabajo conjunto en búsqueda de agenciamiento, teniendo presente las directrices organizacionales, procesando, según el entorno, la información disponible en búsqueda del tipo de decisión más apropiada a la hora de explotar o explorar nuevas alternativas con el fin de superar el evento deconstructor y generar una estrategia emergente, basada en cuasi trayectorias tecnológicas que mantenga la viabilidad de la colección para el cliente.

\section{CONCLUSIONES}

De esta investigación se puede concluir que, dentro del proceso de desarrollo de nuevo producto, soportado en la integración interfuncional, se observan elementos que están relacionados con el momento de la decisión desde la perspectiva de las estrategias deliberadas y emergentes y demás constructos que son considerados importantes dentro de este proceso.

Además, esta aproximación organizacional permite relacionar puntualmente a los conceptos anteriores en el contexto del momento de la decisión a elementos claves como la cuasi trayectoria tecnológica, encuentros, decisiones de exploración y explotación, evento deconstructor, agenciamiento, trabajo conjunto, directrices organizacionales, información y entorno. Donde todos los anteriores, algunos más que otros, se hicieron presentes en esta investigación.

Con este caso de estudio se afianza la idea de que el momento de la decisión está constituido por la relación entre las estrategias deliberadas y emergentes, donde en el medio intervienen los eventos deconstructores que tienen la capacidad de ponerle fin a las estrategias deliberadas y generar la necesidad del surgimiento de estrategias emergentes, denotando el instante en el cual se deben tomar las decisiones.

Finalmente se puede plantear, a manera de hipótesis, que el proceso de desarrollo de nuevo producto está vinculado fuertemente con el grupo de variables descritas anteriormente y que se podría esbozar una especie de estructura que permite caracterizar el proceso y volverlo más efectivo, dependiendo de las condiciones de esas variables. Será tarea de investigaciones futuras dar forma a esa estructura y definir la participación de las variables. 


\section{CONFLICTOS DE INTERÉS}

Los autores declaran que no presentan conflictos de interés financiero, profesional o personal que pueda influir de forma inapropiada en los resultados obtenidos o en las interpretaciones propuestas.

\section{CONTRIBUCIÓN DE AUTORES}

Para el desarrollo de este proyecto, todos los autores han realizado una contribución significativa, especificada a continuación:

Juan Esteban Hernández-Betancur: conceptualización, diseño, desarrollo de la investigación, redacción y revisión del manuscrito.

Luz Alexandra Montoya-Restrepo: conceptualización, diseño, desarrollo de la investigación, redacción y revisión del manuscrito.

Iván Alonso Montoya-Restrepo: conceptualización, diseño, desarrollo de la investigación, redacción y revisión del manuscrito.

\section{REFERENCIAS}

Adamuthe, A. C., Thampi, G. T. (2019). Technology forecasting: A case study of computational technologies. Technological Forecasting and Social Change, v. 143, 181-189. https://doi.org/10.1016/i.techfore.2019.03.002

Becerril-Elías, J. C., Merritt, H. (2021). Alianzas para la innovación en organizaciones intensivas en conocimiento: el caso de México. Revista CEA, v. 7, n. 14, e1780. https://doi.org/10.22430/24223182.1780

Berumen, S. A., Llamazares Redondo, F. (2007). La utilidad de los métodos de decisión multicriterio (como el AHP) en un entorno de competitividad creciente. Cuadernos de Administración, v. 20, n. 34, 65-87. URL

Brown, S. L., Eisenhardt, K. M. (1995). Product development: Past research, present findings, and future directions. Academy of Management, v. 20, n. 2, 343-378. https://doi.org/10.5465/amr.1995.9507312922

Campos y Covarrubias, G., Lule Martínez, N. E. (2013). La observación, un método para el estudio de la realidad. Xihmai, v. 7, n. 13. https://doi.org/10.37646/xihmai.v7i13.202

Chen, L. -H., Tu, C. -C (2014). Time-validating-based Atanassov's intuitionistic fuzzy decision making. IEEE Transactions on Fuzzy Systems, v. 23, n. 4, 743-756. https://doi.org/10.1109/TFUZZ.2014.2327989 
Citroen, C. L. (2011). The role of information in strategic decision-making. International journal of information management, v. 31, n. 6, 493-501. https://doi.org/10.1016/j.ijinfomgt.2011.02.005

Cooper, R. G., Kleinschmidt, E. J. (1986). An investigation into the new product process: Steps, deficiencies, and impact. Journal of Product Innovation Management, v. 3, n. 2, 71-85. https://doi.org/10.1016/0737-6782(86)90030-5

Demory, B. (2007). Como dirigir y animar reuniones de trabajo. Deusto.

Eisenhardt, K. M. (1989). Building Theories from Case Study Research. The Academy of Management Review, v. 14, n. 4, 532-550. https://doi.org/10.2307/258557

Ernst, H., Hoyer, W. D., Rübsaamen, C. (2010). Sales, marketing, and research-and- development cooperation across new product development stages: Implications for Success. Journal of Marketing, v. 74, n. 5, 80-92. https://doi.org/10.1509/jmkg.74.5.080

Genç, E., Di Benedetto, C. A. (2015). Cross-functional integration in the sustainable new product development process: The role of the environmental specialist. Industrial Marketing Management, v. 50, 150-161. https://doi.org/10.1016/j.indmarman.2015.05.001

Good, D. (2014). Predicting real-time adaptive performance in a dynamic decision-making context. Journal of Management and Organization, v. 20, n. 6, 715-732. https://doi.org/10.1017/jmo.2014.54

Greenstein, G. (2015). Timing and decision making. En Euro Working Group Workshop Conferences on Decision Support Systems, EWG-DSS 2014, 89-100. URL

Guo, L., Tobias, J., Bendoly, E., Hu, Y. (2017). Different departments, different drivers: Asymmetry in antecedents and outcomes of voluntary knowledge exchange between sales and production functions. International Journal of Operations \& Production Management, v. 37, n. 8, 1031-1053. https://doi.org/10.1108/IJOPM-01-2016-0046

Helsing, D. (2007). Style of knowing regarding uncertainties. Curriculum Inquiry, v. 37, n. 1, 3370. https://doi.org/10.1111/j.1467-873X.2007.00369.x

Heredia, J. M. (2014). Dispositivos y/o Agenciamientos. Contrastes. Revista Internacional De Filosofía, v. 19, n. 1, 83-101. URL

Hernández Betancur, J. E., Montoya Restrepo, I. A., Montoya Restrepo, L. A. (2016). Variables sustantivas e instrumentales del momento de la decisión. Revista Espacios, v. 37, n. 13. $\underline{U R L}$ 
Hernández-Betancur, J., Montoya-Restrepo, L. A., Montoya-Restrepo, I. (2019). Deliberate strategy deconstructing event for the arising of the emergent strategy. Journal of Engineering and Applied Sciences, v. 14, n. 22, 8452-8463. URL

Jasso Villazul, J. (2004). Trayectoria tecnológica y ciclo de vida de las empresas: una interpretación metodológica acerca del rumbo de la innovación. Contaduría y Administración, n. 214, 84-96. http://dx.doi.org/10.22201/fca.24488410e.2004.469

Jørgensen, J., Mintzberg, H. (1987). Emergent strategy for public policy. Canadian Public Administration, v. 30, n. 2, 214-229. https://doi.org/10.1111/j.1754-7121.1987.tb00079.x

Kotler, P., Keller, K. (2012). Dirección De Marketing (14a ed.). Pearson.

Marion, T. J., Fixson, S. K. (2021). The transformation of the innovation process: How digital tools are changing work, collaboration, and organizations in new product development. Journal of Product Innovation Management, v. 38, n. 1, 192-215.

https://doi.org/10.1111/ipim.12547

Maritz, R., Pretorius, M., Plant, K. (2011). Exploring the interface between strategy-making and responsible leadership. Journal of Business Ethics, v. 98, 101-113. https://doi.org/10.1007/s10551-011-1024-5

Martínez Carazo, P. C. (2006). El método de estudio de caso: Estrategia metodológica de la investigación científica. Pensamiento y Gestión, n. 20, 165-193. URL

Minguela Rata, B., Arias Aranda, D., Rodríguez Duarte, A. (2000). Desarrollo de nuevos productos: consideraciones sobre la integración funcional. Cuadernos de Estudios Empresariales, n. 10, 165-184. URL

Mintzberg, H. (1978). Patterns in strategy formation. Management Science, v. 24, n. 9, 877-975. https://doi.org/10.1287/mnsc.24.9.934

Mintzberg, H. (1987). The strategy concept I: Five Ps for strategy. California Management Review, v. 30, n. 1, 11-24. https://doi.org/10.2307/41165263

Mintzberg, H. (1994). The fall and rise of strategic planning. Harvard Business Review. $\underline{\text { URL}}$

Mintzberg, H., McHugh, A. (1985). Strategy formation in an adhocracy. Administrative Science Quarterly, v. 30, n. 2, 160-197. https://doi.org/10.2307/2393104

Mintzberg, H., Van der Heyden, L. (2006). Revisando el concepto de organización. Harvard Deusto Business Review, n. 150. URL

Mintzberg, H., Waters, J. (1985). Of strategies, deliberate and emergent. Strategic Management Journal, v. 6, n. 3, 257-272. https://doi.org/10.1002/smj.4250060306 
Montoya Restrepo, I. A. (2010). Una contribución a la comprensión de las estrategias deliberadas y emergentes de las organizaciones, desde una perspectiva evolutiva [Tesis de doctorado, Universidad Nacional de Colombia]. https://repositorio.unal.edu.co/handle/unal/70008

Montoya Restrepo, I. A., Montoya Restrepo, L. A. (2013). La formación de estrategias deliberadas y emergentes: Una propuesta a partir de definiciones básicas de una metodología de sistemas suaves. Revista Facultad de Ciencias Económicas, v. 21, n. 2, 67-96.

https://doi.org/10.18359/rfce.657

Muñoz Seco, E., Duro Robles, R., Peguero Rodriguez, E. (2018). ¿Cómo mejorar las reuniones de un equipo de trabajo? FMC - Formación Médica Continuada En Atención Primaria, v. 25, n. 7, 390-396. https://doi.org/10.1016/i.fmc.2017.11.006

Paul, S., Saunders, C. S., Haseman, W. D. (2005). A question of timing: The impact of information acquisition on group decision making. Information Resources Management Journal, v. 18, n. 4, 81-99. https://doi.org/10.4018/irmj.2005100105

Robbins, S. P., Coulter, M. (2005). Administración (8a ed.). Pearson Educación.

Saavedra Mayorga, J. J. (2006). El Tiempo y la Decisión en la Organización desde una Perspectiva Cultural. Revista Facultad de Ciencias Económicas, v. 14, n. 1, 44-65. URL

Sáez Vacas, F., García, O., Palao, J., Rojo, P. (2003). Teoría general del entorno. En Innovación tecnológica en las empresas (1-25). Universidad Politécnica de Madrid.

Sanabria Rangel, M. (2003). La temporalidad de la decisión, la crisis y la construcción de la realidad organizacional. INNOVAR. Revista de Ciencias Administrativas y Sociales, n. 22, 7384.

Senge, P. (1995). La Quinta disciplina. Granica.

Smida, A. (2006). Les moments de decision strategique. Un essai de conceptualisation et de modelisation. Xvème Conférence Internationale de Management Stratégique, Annecy/Genève. $\underline{\text { URL }}$

Song, X. M., Parry, M. E. (1997). The determinants of japanese new product successes. Journal of Marketing Research, v. 34, n. 1, 64-76. https://doi.org/10.1177/002224379703400106

Superintendencia de Sociedades. (2019a). Comportamiento de las 1.000 empresas más grandes del sector real, por ingresos operacionales. $\underline{U R L}$

Superintendencia de Sociedades. (2019b). Informe de las 9000 siguientes empresas más grandes. $\underline{U R L}$ 
Tyagi, S., Kumar, D., Kumar, S. (2019). Reliability based solution to the decision making dilemma in a software environment. Journal of Statistics and Management Systems, v. 22, n. 4, 627634. https://doi.org/10.1080/09720510.2019.1611226

Tzovara, A., Murray, M. M., Bourdaud, N., Chavarriaga, R., Millán, J. D. R., De Lucia, M. (2012). The timing of exploratory decision-making revealed by single-trial topographic EEGanalyses. Neurolmage, v. 60, n. 4, 1959-1969. https://doi.org/10.1016/j.neuroimage.2012.01.136

Urban, G., Hauser, J. (1993). Design and marketing of new products (2da ed.). Prentice-Hall.

Yin, R. K. (2009). Case study Research: Disign and methods (4ta ed.). Sage. https://doi.org/10.33524/cjar.v14i1.73

Yongsheng, Z., Jinjie, X. (2019). The concave relevance between R\&D/Marketing functional integration and product innovation performance of environmentally friendly enterprises in China. Ekoloji, v. 28, n. 107, 1495-1502.

Zanazzi, J. L., Autran, L. F. (2009). La búsqueda de acuerdos en equipos de trabajo: el método decisión con reducción de la variabilidad (DRV). Pesquisa Operacional, v. 29, n. 1, 195-221. https://doi.org/10.1590/S0101-74382009000100010

Zhang, J.-L., Bei, H. (2006). Time pacing: Strategic decision-making for time-based competition. 2006 International Conference on Management Science and Engineering, ICMSE'06 (13 $\left.{ }^{\text {th }}\right)$. https://doi.org/10.1109/ICMSE.2006.314001 


\section{ANEXO 1. Preguntas moderadoras de la entrevista del caso de estudio}

\begin{tabular}{|c|c|}
\hline Constructo & Preguntas del caso \\
\hline Estrategia deliberada y emergente & ¿Qué actores participan en esa toma de decisiones? \\
\hline $\begin{array}{l}\text { Integración interfuncional como estrategia } \\
\text { emergente para la generación de ideas dentro } \\
\text { del proceso de desarrollo de nuevo producto }\end{array}$ & $\begin{array}{l}\text { ¿Existen características particulares de comportamiento } \\
\text { de los actores? }\end{array}$ \\
\hline Momento de la decisión & ¿Cuál es el resultado de la interacción entre los actores? \\
\hline Cuasi Trayectoria tecnológica & $\begin{array}{l}\text { ¿Existe alguna restricción temporal de la acción de cada } \\
\text { uno de los actores? Calendarios o cronogramas }\end{array}$ \\
\hline & ¿Cómo es el proceso de elección de una idea? \\
\hline $\begin{array}{l}\text { Directriz organizacional (cantidad de } \\
\text { alternativas) }\end{array}$ & $\begin{array}{l}\text { ¿Existen directrices gerenciales o del grupo directivo } \\
\text { frente a la generación de ideas? ¿Cuál es efecto? ¿Pueden } \\
\text { sentirse restringidos? }\end{array}$ \\
\hline $\begin{array}{l}\text { Dos agentes por cada departamento (trabajo } \\
\text { conjunto) }\end{array}$ & $\begin{array}{l}\text { ¿De los departamentos que participan en el proceso de } \\
\text { desarrollo de la idea se involucran con una o varios } \\
\text { integrantes del departamento? }\end{array}$ \\
\hline & $\begin{array}{l}\text { ¿Han tenido desarrollos de productos con innovaciones } \\
\text { en donde deban recolectar toda la información } \\
\text { requerida? }\end{array}$ \\
\hline $\begin{array}{l}\text { Disponibilidad de información previa al } \\
\text { encuentro }\end{array}$ & $\begin{array}{l}\text { ¿Qué diferencia se evidencia frente al proceso de toma } \\
\text { de decisiones cuando existe información previa y cuando } \\
\text { no, con respecto al proceso de desarrollo de nuevo } \\
\text { producto? }\end{array}$ \\
\hline $\begin{array}{l}\text { Cantidad de decisiones de exploración y } \\
\text { explotación }\end{array}$ & $\begin{array}{l}\text { ¿La información previa invita a explotar o a explorar } \\
\text { nuevas alternativas? }\end{array}$ \\
\hline Disponibilidad al encuentro (vecinos) & $\begin{array}{l}\text { ¿Qué estrategias aplican para garantizar que los } \\
\text { diferentes departamentos interactúen entre sí en el } \\
\text { marco del proceso de desarrollo de nuevo producto? }\end{array}$ \\
\hline Entorno & $\begin{array}{l}\text { ¿Existe vigilancia del entorno para identificar elementos } \\
\text { que afecte el proceso de toma de decisiones? ¿Tienen } \\
\text { identificados algunos? }\end{array}$ \\
\hline Evento deconstructor & $\begin{array}{l}\text { ¿Han afrontado situaciones dentro de la elección de la } \\
\text { idea que deshace lo planeado? ¿Algunas veces esas } \\
\text { situaciones los llevan a ideas viables y que no habían sido } \\
\text { consideradas? }\end{array}$ \\
\hline $\begin{array}{l}\text { Agenciamiento (dos departamentos con el } \\
\text { mismo comportamiento) }\end{array}$ & $\begin{array}{l}\text { ¿Dentro del proceso interdisciplinario de la generación de } \\
\text { ideas se identifica que hay departamentos que } \\
\text { potencializan ideas por tener afinidad con ciertas } \\
\text { alternativas? ¿Qué efecto tiene este tipo de alianzas? }\end{array}$ \\
\hline
\end{tabular}

Fuente: elaboración propia. 